\title{
BMJ Open Advance Care Planning: Promoting Effective and Aligned Communication in the Elderly (ACP-PEACE): the study protocol for a pragmatic stepped-wedge trial of older patients with cancer
}

\author{
Joshua R Lakin (D) , ${ }^{1,2}$ Elise N Brannen, ${ }^{1}$ James A Tulsky, ${ }^{1,2}$ \\ Michael K Paasche-Orlow, ${ }^{3}$ Charlotta Lindvall, ${ }^{1,2}$ Yuchiao Chang, ${ }^{2,4}$ \\ Daniel A Gundersen, ${ }^{5}$ Areej El-Jawahri, ${ }^{2,6}$ Angelo Volandes, ${ }^{2,4}$ The ACP-PEACE \\ Investigators
}

To cite: Lakin JR, Brannen EN, Tulsky JA, et al. Advance Care Planning: Promoting Effective and Aligned Communication in the Elderly (ACP-PEACE): the study protocol for a pragmatic stepped-wedge trial of older patients with cancer. BMJ Open 2020;10:e040999. doi:10.1136/ bmjopen-2020-040999

- Prepublication history and additional material for this paper are available online. To view these files, please visit the journal online (http://dx.doi. org/10.1136/bmjopen-2020040999).

Received 27 May 2020 Revised 22 June 2020 Accepted 30 June 2020

Check for updates

(C) Author(s) (or their employer(s)) 2020. Re-use permitted under CC BY-NC. No commercial re-use. See rights and permissions. Published by BMJ.

For numbered affiliations see end of article.

Correspondence to

Dr Joshua R Lakin;

jlakin@partners.org

\section{ABSTRACT}

Introduction Advance care planning (ACP) is associated with improved health outcomes for patients with cancer, and its absence is associated with unfavourable outcomes for patients and their caregivers. However, older adults do not complete ACP at expected rates due to patient and clinician barriers. We present the original design, methods and rationale for a trial aimed at improving ACP for older patients with advanced cancer and the modified protocol in response to changes brought by the COVID-19 pandemic.

Methods and analysis The Advance Care Planning: Promoting Effective and Aligned Communication in the Elderly study is a pragmatic, stepped-wedge cluster randomised trial examining a Comprehensive ACP Program. The programme combines two complementary evidence-based interventions: clinician communication skills training (VitalTalk) and patient video decision aids (ACP Decisions). We will implement the programme at 36 oncology clinics across three unique US health systems. Our primary outcome is the proportion of eligible patients with ACP documentation completed in the electronic health record. Our secondary outcomes include resuscitation preferences, palliative care consultations, death, hospice use and final cancer-directed therapy. From a subset of our patient population, we will collect surveys and video-based declarations of goals and preferences. We estimate 11000 patients from the three sites will be enrolled in the study. Ethics and dissemination Regulatory and ethical aspects of this trial include Institutional Review Board (IRB) approval via single IRB of record mechanism at DanaFarber Cancer Institute, Data Use Agreements among partners and a Data Safety and Monitoring Board. We plan to present findings at national meetings and publish the results.

Trial registration number NCT03609177; Pre-results.

\section{INTRODUCTION}

More than half of newly diagnosed malignancies occur in patients over the age of 65

\section{Strengths and limitations of this study}

- The strengths of this study lie in its pragmatic design, allowing for 'real world' evidence for two interventions that have been previously tested in more controlled settings.

- The stepped-wedge design is practical and considered the design of choice when it is logistically impractical to simultaneously roll out the intervention to half of the clusters.

- The biggest limitation we are currently facing has to do with the possible notable change in secular trends due to the COVID-19 and the impact that it has on advance care planning. To address this issue, we have adjusted our analysis plan to account for these changes.

- We are limited by the quality of structured and present variables in the electronic health records of each site, especially for advance care planning; however, our use of natural language processing helps to rectify for lack in accuracy.

- In addition to the above change in secular trends due to COVID-19, this trial design can be affected by ongoing innovation in cancer care delivery, such as the continuing growth of immunotherapy changing prognosis for some of these advanced cancers in significant ways, thus affecting our results.

years $^{1}$ and that same population accounts for over two-thirds of all adult US cancer deaths. ${ }^{2}$ In addition to high mortality, older adults with cancer suffer disproportionately from receiving medical interventions that do not reflect their values and preferences. ${ }^{3-5}$ Advance care planning (ACP) seeks to align medical care with patients' values and preferences. ${ }^{67} \mathrm{ACP}$ is consistently associated with better outcomes, ${ }^{89}$ while a lack of ACP is associated with greater use of unwanted medical 
interventions, more terminal hospitalisations, lower hospice use, higher healthcare costs and worse bereavement outcomes. ${ }^{30-14}$ Despite evidence supporting ACP, participation rates remain low among older adults with serious illness, such as cancer. ${ }^{15}$

Effective ACP requires that patients experience accurate and comprehensible communication early in their illness, ${ }^{14} 16-18$ a collaborative effort requiring education for both patients and clinicians. Unfortunately, studies suggest that traditional written ACP can be ineffective in sufficiently informing patients and often occurs late in the disease process, ${ }^{19-24}$ with the risk that patients' understanding is clouded by pain, medication or psychological distress. ${ }^{10} 2025$ The heightened emotional state associated with hearing bad news late in a disease course interferes with patients' cognitive processing, and this reaction may be exacerbated by clinician inattention to affect. ${ }^{21}$ 26-29 Patients assign considerable importance to their physicians' statements regarding ACP and the quality of communication ${ }^{30}$ and while $90 \%$ of patients say they want to talk to their doctors about their stress and concerns, ${ }^{31} 32$ physicians generally, and oncologists specifically, often do not communicate effectively regarding ACP and end of life. $^{30}$ 32-38 Therefore, an effective intervention should both prepare patients for shared decision making and improve clinicians' communication skills.

We have developed a comprehensive ACP programme to drive improved communication and ACP for an ageing US cancer population using a combination of empirically proven patient video decision aids and clinician communication skills training. This programme integrates video decision aids for patients (ACP Decisions) and a clinician communication training programme (VitalTalk) into 12 disease-based oncology clinics each across three health systems with the aim of improving conversations and documentation of ACP. By providing both patients and clinicians with the necessary tools and training, we create an inclusive approach to optimise ACP before the toughest choices arise for patients.
Most trials targeting older patients with serious illness evaluate interventions under ideal conditions and involve few facilities. ${ }^{39-42}$ Thus, we need research for this population using pragmatic trials. ${ }^{43}$ We sought to test this intervention in a manner that allows for improvements in processes as we learn them. ${ }^{44}$ Advance Care Planning: Promoting Effective and Aligned Communication in the Elderly (ACP-PEACE) is a pragmatic stepped-wedge cluster randomised trial (SW-CRT) that conducts a realworld test of the comprehensive ACP programme in older patients with cancer. In this paper, we present the design, methodology and rationale for the ACP-PEACE trial and discuss our adjustments for the novel coronavirus COVID-19 19 pandemic.

\section{METHODS \\ Overview}

We are studying the combination of clinician training and patient videos via a pragmatic SW-CRT and analysing electronic health records (EHRs) for ACP outcomes for patients aged 65 years and older. Using small subsamples of patients, we will also assess patient-centred outcomes using surveys and video declarations in which patients discuss their values and preferences in their own words on video (figure 1). We used the Standard Protocol Items: Recommendations for Interventional Trials reporting guidelines for this manuscript. ${ }^{45}$

\section{Study timeline}

The ACP-PEACE study has two phases, a characteristic of the funding mechanism. The UG3 phase (year 1) of the study focused on developing and refining the intervention and data acquisition. In this phase, we established our organisational structure, developed the processes and infrastructure needed to conduct the trial and pilottested the study intervention in three clinics, one from each participating health system. During the UH3 phase (years 2-5), we planned to introduce the intervention to

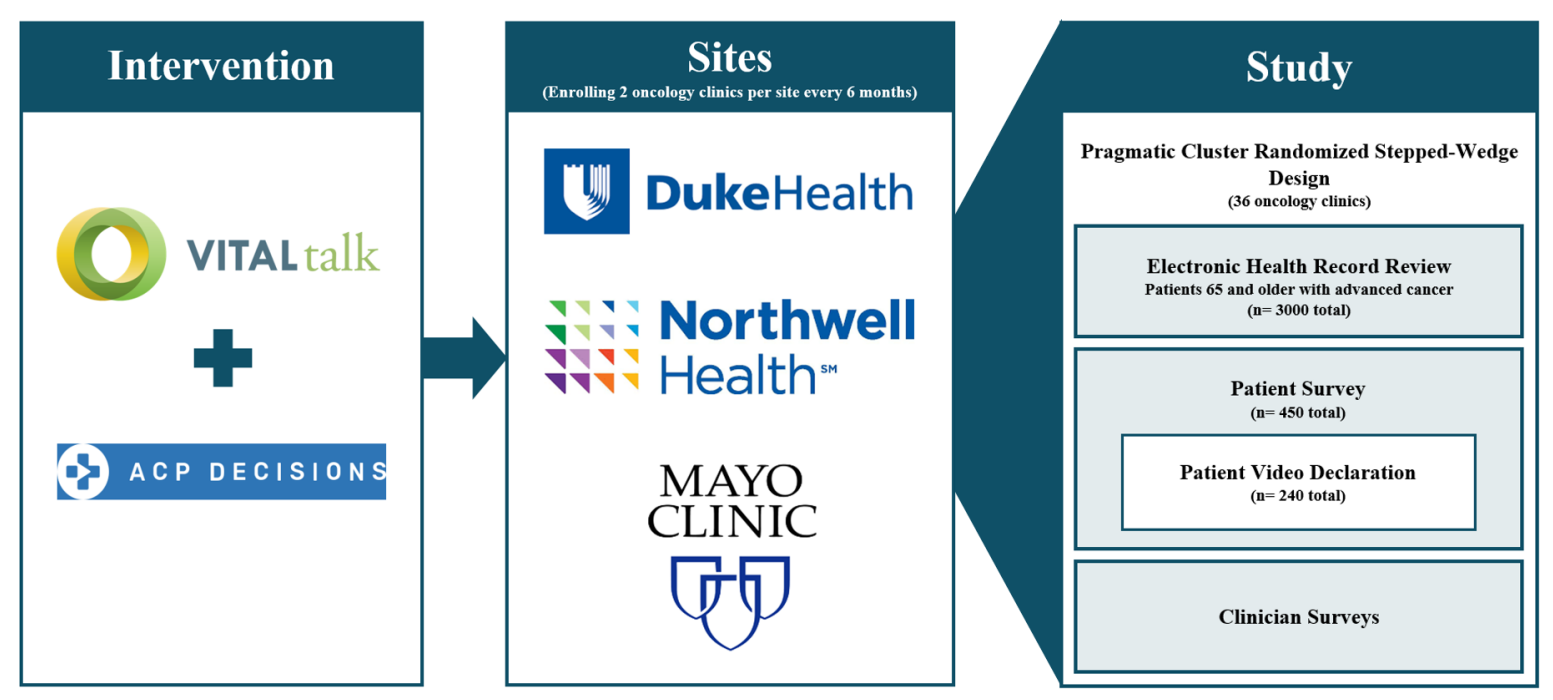

Figure 1 Advance Care Planning: Promoting Effective and Aligned Communication in the Elderly model. 


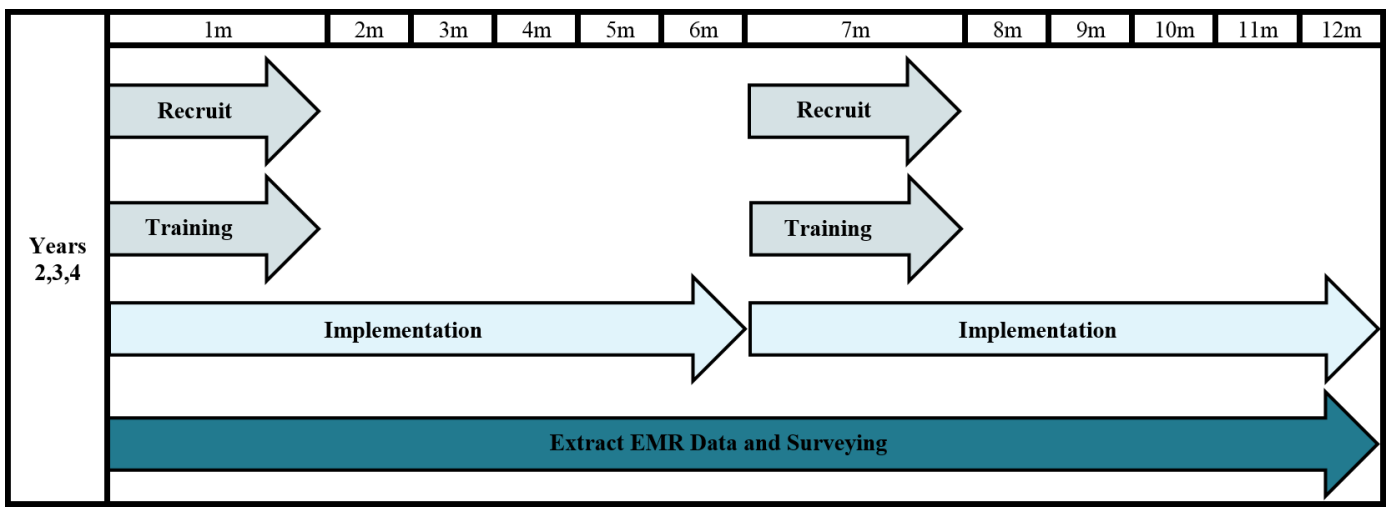

Figure 2 Stepped-wedge recruitment and implementation yearly timeline (repeated each year). EMR, Electronic Medical Record.

the 36 remaining oncology clinics in 6-month waves: two clinics per system for a total of six clinics every 6 months (figure 2).

\section{Sites and randomisation}

We will draw participants from disease-based oncology clinics from three unique systems: Duke Health (North Carolina), Mayo Clinic (Minnesota) and Northwell Health (New York). These sites are geographically, socioeconomically and culturally distinct. Each participating clinic has more than one practising oncologist, and to be eligible for randomisation, at least $30 \%$ of the patient population must be aged 65 years or older.

For the UH3 phase, we have identified a total of 36 oncology clinics (12 per site) as candidate clinics based on recent data from each system. The pilot clinics that participated in the UG3 phase tested the intervention process and will not be included in the final analysis. In the UH3 phase, we will use stepped-wedge cluster randomisation with the clinic as the unit of randomisation. With the clinic as the unit of randomisation, we avoid the contamination that can occur when randomising individuals within each clinic. The sequence of randomisation was generated prior to initiation of the trial via random number generator. Every 6 months after the baseline, two clinics from each system will be randomised to the intervention (figure $3 \mathrm{~A}$ ).

During the original Step 2, COVID-19 spread throughout the country interrupting the stepped-wedge design in two key respects: (1) the team was unable to conduct the in-person trainings for the step 2 intervention clinics; and (2) ACP activities are likely to increase during this period due to a response to the pandemic, irrespective of the study. On the recommendation of the NIH Collaboratory Statistics Core, we modified the original design to 'restart' the trial for the remaining 30 clinics using the original step 2 as the new baseline. The training of the remaining 30 control clinics will be over four steps to keep the trial completion on the same overall timeline (figure 3B).

\section{Population}

We will evaluate the outcomes for patients aged 65 years or older with advanced cancer across all 36 clinics. As the intervention will be implemented clinic wide, rather than targeted to specific study patients, all intervention clinic patients can receive the intervention. We will analyse data for patients with advanced cancer aged 65 years or older; patients' data will be counted towards control or intervention based on the allocation of each clinic at the end of each period of the stepped-wedge design. Therefore, a given patient could contribute data during more than one period and could contribute data to both control and intervention periods.

During the UH3 years, research assistants at each site will conduct in-person surveys with 450 randomly selected patients (150 per site) for our secondary exploratory patient-centred outcomes. Patients selected for surveys will be distributed evenly among clinics within each system and will include an equal number of surveys of patients from clinics in the control and intervention phases. Patients will be surveyed only once as patients surveyed in the control phase will be excluded from completing the later intervention survey. Additionally, from among this group of 450 surveyed patients, a subgroup of 240 will be randomly selected and asked to conduct a video declaration activity. All patients selected for surveys or videos will be excluded from the primary study population to avoid bias rendered from additional contact with the study team.

\section{Intervention design, implementation and adherence monitoring}

The comprehensive ACP intervention combines VitalTalk and ACP Decisions, two evidence-based interventions previously used separately, to create an innovative dual approach to improving ACP. These interventions are complementary, as one targets improvement of clinicians' skills and the other prepares patients for shared decision making. VitalTalk is the most widely disseminated teaching method for effective communication skills training based on practice and feedback on one's own communication skills. Supported by numerous previous 
A

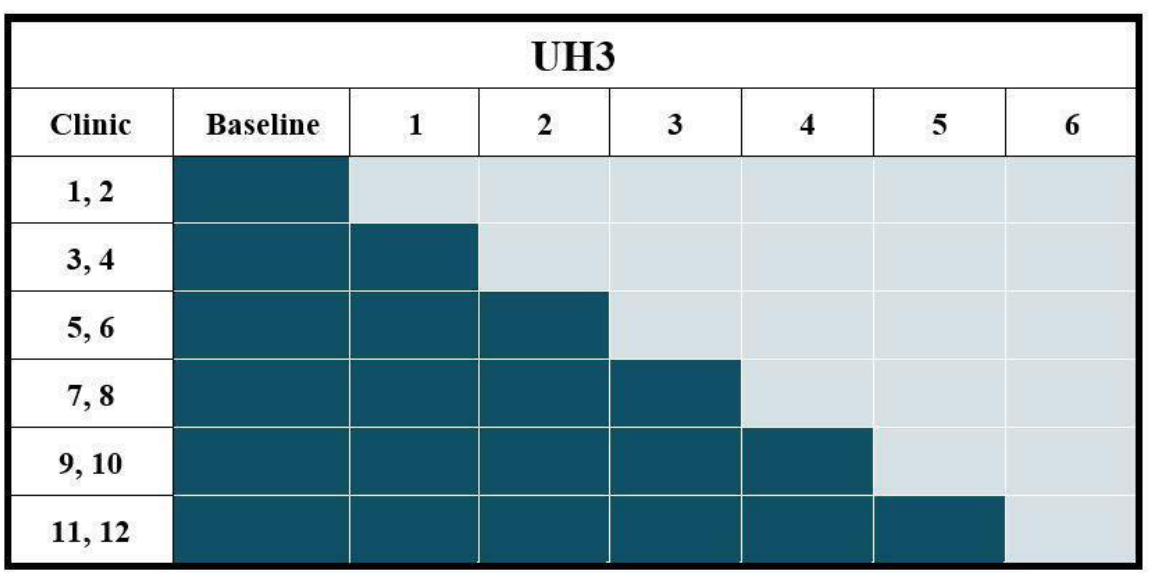

․ Intervention

Control

B

\begin{tabular}{|c|c|c|c|c|c|c|c|c|c|}
\hline Clinic & Baseline & 1 & 2 & 3 & 4 & 5 & 6 & Intervention & Covid-19 \\
\hline 1,2 & & & & & & & & No & Pre \\
\hline & & & Baseline & 1 & 2 & 3 & 4 & No & Post \\
\hline $3,4,5$ & & & & & & & & Yes & Pre \\
\hline 6,7 & & & & & & & & Yes & Post \\
\hline $8,9,10$ & & & & & & & & & \\
\hline 11,12 & & & & & & & & & \\
\hline
\end{tabular}

Figure 3 (A) Original stepped-wedge cluster randomisation scheme within each health care system. (B) Modified steppedwedge cluster randomisation scheme within each health care system.

studies, ${ }^{46-52}$ VitalTalk leverages didactics, demonstration and small group sessions using role play with trained actors portraying patients through which clinicians learn effective delivery of serious news, prognosis discussion and early and late goals-of-care conversations. For this study, the VitalTalk course will be a half-day session that teaches a framework for late goals-of-care discussions, including skills around delivery of serious news, responding to emotion, assessing prognostic awareness, identifying what is most important to patients and making recommendations.

The ACP Decisions programme uses short video decision aids to address the most common issues facing older patients with serious illness. Videos in over 25 languages can be prescribed to patients and caregivers and are easily accessed in a mobile app or through a web-based platform. The ACP Decisions videos have been shown to increase knowledge, decision certainty and the stability of preferences over time and to better inform the way that patients choose healthcare interventions towards the end of life.$^{53-72}$ The video collection includes certified video decision aids, ${ }^{73}$ regarding ACP, advance directives, healthcare agents, goals of care, cardiopulmonary resuscitation and hospice, that have been studied in a statewide implementation showing greater patient-aligned medical care. $^{72}$

We will provide in-person training every 6 months at each new clinic added to the intervention period of the trial. The comprehensive ACP training programme uses the VitalTalk methodology and infrastructure and the ACP Decisions Program tools to instruct clinicians and staff on how to: (1) more effectively communicate with patients with cancer, (2) have ACP conversations with patients, (3) introduce the videos to patients and families, (4) use the videos as an adjunct to ACP counselling by clinicians, (5) select the appropriate video(s) according to patient needs and (6) use the application or electronic platform for viewing videos. The combined programme will involve a half-day face-to-face joint VitalTalk and ACP Decisions training. Any staff member affiliated with the selected facilities will be eligible to participate in training. As staff turnover among the sites is expected, training will be made available on an ongoing basis throughout the trial.

Immediately following the initial training at each site, we will deploy the remainder of the intervention infrastructure. The ACP Decisions videos will be programmed into desktop devices, tablets and password-protected 
electronic platforms of each health system's intranet. When clinics initiate the intervention, they will implement the videos with all patients with flexibility as to which providers (physician, nurse and social worker) introduce the videos and exactly which videos are used to meet their patients' clinical needs. Additionally, the in-person clinician training will be supplemented with emails, pocket cards, offers of coaching and online educational videos. The study team will facilitate dissemination of implementation successes and challenges via a learning network by conducting 1-hour webinars at each of the practices randomised to the intervention every other month to discuss quality improvement activities relating to the study. The intensity of the VitalTalk training implementation will be assessed as the proportion of eligible staff trained, including new staff joining the practice over the implementation period. The intensity of implementation of the ACP Decisions videos will be assessed as the ratio of the number of videos viewed using the site-specific access codes captured at the ACP Decisions website to the number of eligible patients at each site for each 6-month intervention period. Fidelity to the video component of the intervention will be monitored by tracking of video use (which videos are used at each clinic, playthrough rate and frequency). Feedback on video viewing will be shared with each site at the end of each 6-month implementation phase. Last, we aim to evaluate the impact of the study with a novel video declaration process, allowing patients to state their values and preferences in their own terms, which is described in detail in the online supplementary appendix. ${ }^{59}$

\section{Control condition}

Clinics in the control phase will use whatever ACP procedures already exist in place at their respective system. Although current ACP improvement initiatives may be present and vary from clinic to clinic, this heterogeneity reflects the current dynamic state of 'usual' care and is therefore appropriate in this pragmatic trial. ${ }^{43}$

\section{Outcomes}

The outcomes of the ACP-PEACE trial can be divided into three main categories: patient level, clinician level and system level. Our primary outcome is the proportion of eligible patients with ACP documentation completed in the EHR. Presence of completed ACP documentation will be defined via one or both of the following two means: (1) structured EHR data: scanned forms including advance directives, living wills or Physician's Orders for Life Sustaining Treatment (or state-specific equivalent) and code status orders indicating do not resuscitate status (or similar site-specific codes for limitations on treatments) and (2) Natural Language Processing (NLP) extraction (described below in detail): clinical documentation that will include goals-of-care discussion, ACP, hospice discussion, discussion of palliative care or limitations on code status. From the EHR or the local tumour registry, we are also determining demographic covariates and baseline data. Secondary outcomes include resuscitation preferences, palliative care consultations, death, hospice use/ utilisation at the end of life and final cancer-directed therapy.

We are deriving patient-centred outcomes from the patient survey and video declarations. The surveys measure our patient-centred secondary outcomes such as patient confidence that their future medical care will match their values, satisfaction with their clinicians' communication, ${ }^{74} 75$ satisfaction with their medical deci$\operatorname{sion}^{76}$ and regret about their medical decision (online supplementary appendix). ${ }^{7778}$ Finally, for each of the 450 surveyed patients who die during the study period, we will extract data, via a chart abstraction tool, regarding ACP preferences and care received in the final 3 months of life to explore whether patients receive goal-concordant care.

We are also collecting a small set of clinician data points. Participating clinicians provide information on demographics, clinical experience, prior communication training and socioemotional orientation. ${ }^{52}$ table 1 lists each data element, with its purpose, proposed source and the target population from whom we need the data for successful completion of the study. System-level data measurement will include measurement of the training and video use as described above as well as exploratory analysis of coaching calls and implementation activities.

\section{Data sources, data elements and linkage}

Baseline (ie, preintervention) data for all randomised clinics will include a 6-month period prior to date of intervention delivery. Patients will be identified as having advanced cancer from each site's tumour registry and/ or from clinical ICD codes, which have been studied in some cancers and have demonstrated strong specificities. ${ }^{79}$ While these methods have lower sensitivity, they capture enough patients with advanced cancer with high specificity for outcome assessment without systematic bias towards intervention or control periods. Demographic information and baseline characteristics relevant to general oncology will be collected from the EHR. Our primary and secondary outcomes will be abstracted from the local EHRs and tumour registries as detailed below in outcomes.

We will also use NLP, a form of computer-assisted abstraction, to detect our primary and secondary outcomes. Our NLP software, ClinicalRegex, identifies predefined keywords or phrases within clinical notes, considering varieties in language and punctuation. ${ }^{80-82}$ ClinicalRegex also allows for rapid semiautomated review that ensures that keywords have not been taken out of context. For each NLP process (ie, goals-of-care discussion), we have built a keyword library that identifies relevant documentation within clinical notes. Each keyword library was refined and validated by manual review of clinical notes in local EHRs. With NLP, we will collect additional data on ACP documentation, goals-ofcare discussions, limitation of life-sustaining treatment, palliative care consultation and hospice assessment. 
Table 1 Data elements and outcomes

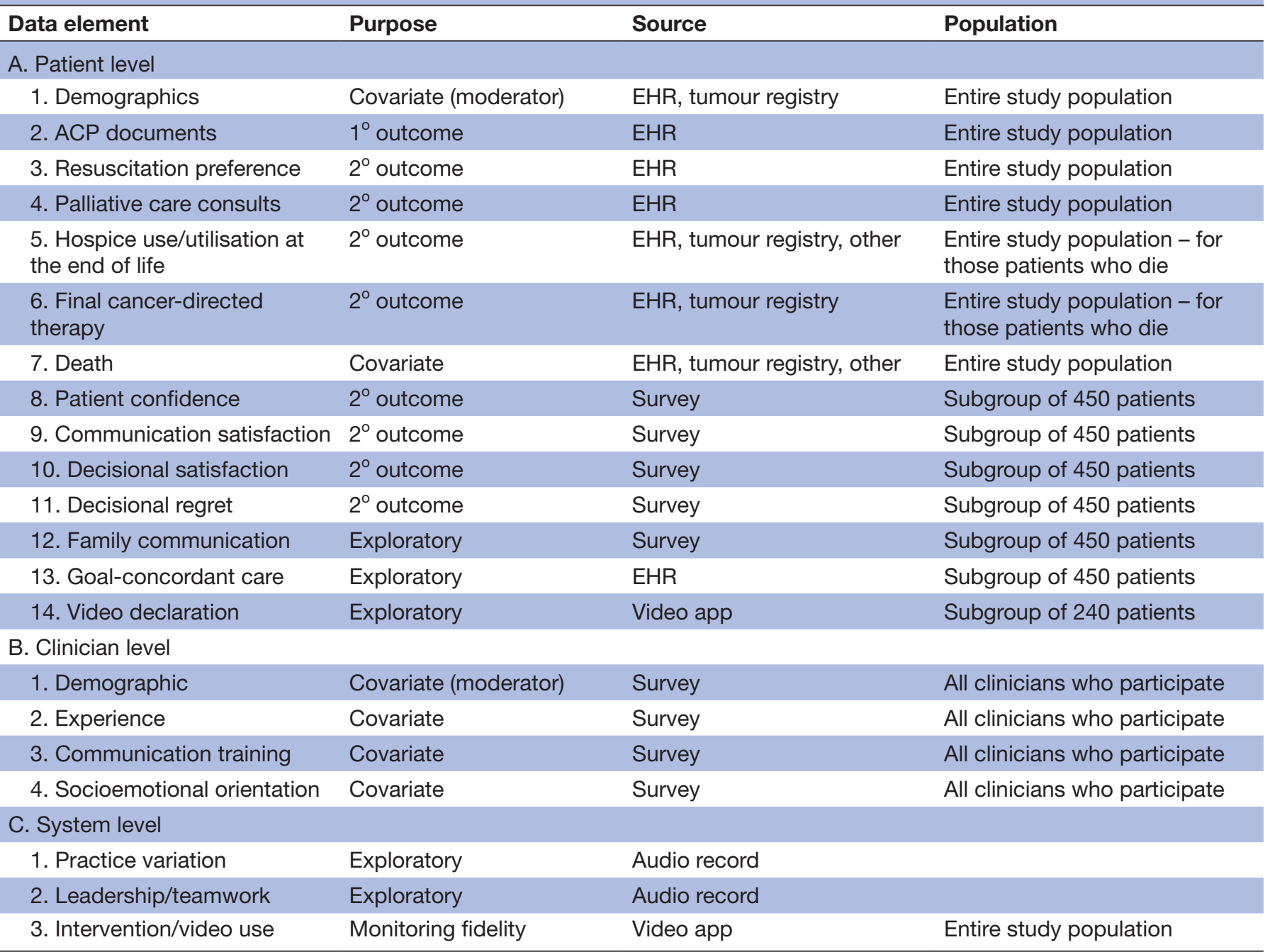

ACP, Advance Care Planning; EHR, electronic health record.

Exploratory patient-centred outcomes and clinician outcomes will be derived from surveys collected through REDCap. ${ }^{83} 84$

Data use agreements between all systems are on file, and each site maintains and adheres to the process and procedures for the protection of human subjects and protected health information (PHI) for their covered entities. Only the minimum amount of necessary PHI will be collected from participants. Health Insurance Portability and Accountability Act-compliant and passwordprotected servers will be used to store all collected data. Individual password-protected files will separate participant identifiers and a third password-protected linking file will be maintained. This linking file has restricted access and uses a logging feature that identifies each user and instance of use. All data will be transmitted via secure methods approved by the respective institutions to the Dana-Farber Cancer Institute (DFCI) for data management and to Boston Medical Center for qualitative analysis, and trial investigators will have access to the final data set, and it will be made available on reasonable request.
The EHR data will undergo a review-adjudication process whereby DFCI data staff and key, unblinded investigators review the raw data for each variable to identify out of range or unexpected values; a summary is sent to each site, and conference calls are conducted with relevant investigators and programmers to adjudicate any issues. We will also validate a randomly selected subset of data, verifying key demographic characteristics and patient selection criteria against medical records. The EHR data are then uploaded to a REDCap database.

\section{Masking}

Blinding for this trial occurs at multiple levels. Research Coinvestigators at each site will be aware of the randomisation order as well as which clinics receive the intervention and when. The investigators leading the trainings will likewise be aware of which clinics receive the intervention. Similarly, due to their roles in working with the data and generating video adherence reports for the intervention clinics, certain members of the implementation and data management teams will be unblinded 
to clinic assignments and outcomes. All other staff will remain blinded to randomisation scheme and outcomes.

\section{Statistical analysis}

Our primary analytical approach uses an intention-to-treat analysis, with no special allowance for non-compliance or non-adherence. With the stepped-wedge design, the outcomes during the intervention (exposed) periods will be compared with outcomes during the control (unexposed) periods. We will conduct two analyses based on the observations included in the analysis: (1) open cohort with repeated measures design: individuals may leave and others may join during the study and the same individuals are allowed to appear in multiple periods, (2) repeated cross-sectional design: subjects will only be included in the period when they first enter the study. Characteristics of the individuals and clusters will be summarised by exposure status. ${ }^{85}$ We will use generalised linear mixed models to compare outcomes between intervention and control periods. The basic model is depicted in this equation:

$$
g\left(Y_{i j k}\right)=\mu+\alpha_{i}+\beta_{j}+\gamma_{k}+X_{i j} \theta
$$

where $Y_{i j k}$ denotes the response from individual $k$ at time $j$ from cluster $i$. To account for clustering within each clinic, the model includes a random effect $\alpha_{i}$ for cluster $i$. Under the stepped-wedge design, calendar time is associated with the exposure to the intervention. We will include a fixed effect $\beta_{j}$ to adjust for potential confounding factors from calendar time. In the case that time effect might not be the same for all clusters, we will change the term from a fixed effect $\beta_{j}$ to a random effect $\beta_{i j}$. To account for repeated measures from the same subject from the first analysis, we will include a random subject effect $\gamma_{k}$. The term $X_{i j}$ represents the treatment indicator in cluster $i$ at time $j$ with $\theta$ representing the overall treatment effect. If there is evidence of treatment effect heterogeneity, we will either change the fixed effect $\theta$ to a random effect $\theta_{i}$ or change the fixed effect $\theta$ to $\theta_{(s)}$, which allows different treatment effects for different strata. We will also explore heterogeneity of intervention effect for different subgroups by adding an interaction term between treatment status and subgroup to the models. These groups include site, sex as a biological variable, race/ethnicity (white vs non-white) and different types of cancer diagnoses.

If necessary, we will include additional terms $\delta_{1} Z_{i j k}$ and $\delta_{2} W_{i j}$ to the model, where $Z$ and $W$ represent vectors of patient and cluster characteristics. The index $j$ in the $Z$ matrices allows us to include the time-varying covariates, which correspond to any patient characteristics that could change over time. We will use a logit link $(g)$ for the binary outcomes that include our primary outcome of ACP documentation and our secondary outcomes of resuscitation preference and hospice use. Other outcomes such as number of palliative care consults and utilisations are considered as Poisson variables and modelled with a $\log$ link.
In adjustment for the COVID-19 19 pandemic, the analysis plan will remain the same for the data collected from the 30 clinics randomised to intervention after the original step 2 (figure 3B). The data collected from the six clinics that received intervention during step 1 will allow us to examine the ACP Programme intervention effect prior to COVID-19 by comparing the ACP rates prior to the intervention (original baseline) and after the intervention (original step 1). Additionally, ACP rates from original baseline, step 1 and step 2 from the 30 clinics randomised to intervention after the original step 2 will be used to estimate the 'COVID-19 effect' on ACP.

We also have patient-centred secondary outcomes from survey results for analysis. Since patients will be surveyed in the step immediately before and after the intervention is initiated within each clinic, the number of intervention and control patients will be approximately equal at each time point. We will use linear mixed models that treat time (ie, before or after intervention) as a fixed effect and clinic as a random effect to account for clustering of patients within clinics.

Finally, we will examine care delivery alignment with expressed goals from a subset of deceased patients of those 450 surveyed. Using a chart abstraction tool, two blinded expert investigators will judge whether patients received care concordant with their documented wishes. Coders will make determinations and discuss disagreements; final judgments will be determined by consensus. For qualitative coding evaluation, we will summarise the extent of agreement using kappa statistics and will compare results between those who died before and after receiving interventions.

\section{Statistical power and sample size requirements}

We used the Hooper et $a l^{868}$ approach to conduct the power analysis. We originally estimated close to 5000 patients from 36 oncology practices are eligible for the study at each time point and approximately $20 \%$ are new patients at each step. With seven time points (baseline plus six steps), we anticipated a total of 11000 unique patients will be included in the study. With the modified design, we estimate 4160 patients from 30 oncology practices are eligible for the study at each time point, and a total of 7500 unique patients will be included in the steppedwedge design analysis. With each clinic contributing an average of 139 patients at each step from the cohort design, the design effect due to clustering is 7.9 assuming an intracluster correlation of 0.05 , and the design effect due to repeated assessment is 0.12 assuming the cluster autocorrelation coefficient is 0.7 and the individual autocorrelation coefficient is 0.9 . These estimates correspond to an effective sample size (ie, sample size required for individual randomization) of 4405 . For the repeated cross-sectional design, each clinic will contribute an average of 23 new patients at each step, and the effective sample size is 1628 with the same assumptions on intracluster correlation and cluster autocorrelation. Preliminary estimates indicate the rate for ACP documentation 
(the primary outcome) ranges from $15 \%$ to $30 \%$ for the control periods, which requires an effective sample size of 500-954 for detecting a 10\% absolute increase in our primary outcome with a two-sided significance level of 0.05 . Therefore, the study will have more than $90 \%$ power for either analysis using the open cohort with repeated measures design or the repeated cross-sectional design.

For the patient-centred survey outcomes, 225 patients will be surveyed during control periods and 225 will be surveyed during intervention periods. Assuming an intraclass correlation coefficient of 0.05 and an average cluster size of 12.5, the effective sample size is approximately 286 . A sample of this size allows for $90 \%$ power to detect a small to moderate effect size (Cohen's $d$ ) of $0.39 \%$ and 99\% power to detect a moderate effect size of 0.5 for outcomes such as patient confidence, decisional satisfaction and regret.

\section{ETHICS AND DISSEMINATION \\ Regulatory considerations}

Regulatory aspects of this trial include Institutional Review Board (IRB) approval, Data Use Agreements among partners and an independent Data Safety and Monitoring Board. This study was approved via a single IRB of record mechanism as a multicentre trial with the DFCI as the lead site. Duke Health, Mayo Clinic and Northwell Health are participatory sites and Boston Medical Center and Massachusetts General Hospital are non-participatory sites. Each site's own regulatory board established official 'reliance agreements' to use the DFCI's Office of Human Research Subjects (OHRS) as their main regulatory agent. The three participating sites have formally designated via SMART IRB that the IRB of record is the DFCI IRB and agree to follow the rules and regulations set forth by the DFCI OHRS. All relevant parties are notified by email of any protocol modifications. This study presents minimal risk to participants. Investigators will monitor and report any unforeseen adverse events to the IRB. We have proactively requested an audit to be conducted by DFCI's OHRS before the trial end. Committees consisting of the various investigators oversee overall project direction and administration, intervention implementation, data quality and monitoring, stakeholder engagement, and regulatory and ethical considerations. Data use agreements between all systems are on file, and each site maintains and adheres to the process and procedures for the protection of human subjects and PHI for their covered entities. Patients will be notified of the study and their participation via broadcast notifications in the form of posters in each of the clinics and will have the option to opt out. A waiver of consent was approved for the EHR review of the primary study subjects who are not contacted by study staff unless a specific research declination is on file at that site. Waivers of consent were also approved for engaging participating clinicians and surveyed patients not completing the video declaration as their participation is confidential and voluntary giving implied consent, and there is minimal risk with the study. Those surveyed patients who also elect to complete the video declaration first need to sign an approved written consent form obtained by RAs at each site.

\section{Relevance and dissemination}

The ACP-PEACE trial will be the first to study combining two evidence-based interventions in a pragmatic setting. The work combines clinician training in responding to emotion and handling difficult conversations with decision video aids for patients. The strengths of the study include the complementary nature of these approaches: targeting both clinicians and patients in a novel way. Additionally, the pragmatic nature of the trial allows us to collect evidence of the effect of these interventions in a 'real-world' setting and provides rich information on the implementation of ACP interventions. This study has the potential to add to a growing literature informing large systematic ways of improving ACP for older adults with cancer. We plan to publish the primary outcome related to ACP documentation and our secondary outcomes in a single paper. We will also perform further analyses of our Natural Language Processing methods, exploratory outcomes, chart review, implementation outcomes and video declarations and present these in publication and at national meetings.

\section{Author affiliations}

${ }^{1}$ Department of Psychosocial Oncology and Palliative Care, Dana Farber Cancer Institute, Boston, Massachusetts, USA

${ }^{2}$ Harvard Medical School, Boston, Massachusetts, USA

${ }^{3}$ Department of General Internal Medicine, Boston University School of Medicine, Boston Medical Center, Boston, Massachusetts, USA

${ }^{4}$ Department of Medicine, Massachusetts General Hospital, Boston, Massachusetts, USA

${ }^{5}$ Department of Survey and Data Management Core, Dana Farber Cancer Institute, Boston, Massachusetts, USA

${ }^{6}$ Department of Hematology-Oncology, Massachusetts General Hospital, Boston, Massachusetts, USA

Twitter Charlotta Lindvall @lindvalllab

Collaborators The ACP-PEACE Investigators: Julie Goldman, MSW, MS; Brian Sipin, BSc; Michael J Barry, MD; Kathryn I Pollak, PhD; Miji Sofela, MBChB; Danielle Kennedy, MPH; S. Yousuf Zafar, MD; Maria Torroella Carney, MD; Diana MartinsWelch, MD; Michael Qiu, MD, PhD; Jody-Ann McLeggon, MPH; Craig E Devoe, MD; Jon C. Tilburt, MD; Charles L Loprinzi, MD; Parvez A. Rahman, MHI; Jeremiah J. Stout, BA; Aretha Delight Davis, MD, JD; and Lisa M. Quintiliani, PhD

Contributors Study concept and design: AV and JAT. Acquisition of data: JRL, ENB $\mathrm{CL}, \mathrm{DAG}$ and The ACP-Peace Investigators. Analysis and interpretation of data: JRL, CL, DAG, YC, JAT, MKP-0, AE-J and AV. Drafting of the manuscript: JRL, ENB, JAT, MKP-0, CL, YC, DAG, AE-J, The ACP-PEACE Investigators and AV. Critical revision of the manuscript for important intellectual content: JRL, ENB and AV. Statistical analysis: YC. Obtained funding: AV and JAT. Administrative, technical or material support: ENB and The ACP-PEACE Investigators. Study supervision: AV and JAT.

Funding Research reported in this publication was supported within the National Institutes of Health (NIH) Health Care Systems Research Collaboratory by cooperative agreement UH3AG060626 from the National Institute on Aging. This work also received logistical and technical support from the NIH Collaboratory Coordinating Center through cooperative agreement U24AT009676.

Disclaimer The content is solely the responsibility of the authors and does not necessarily represent the official views of the National Institutes of Health.

Competing interests JRL receives funding from the Cambia Health Foundation as part of the Sojourns Scholars Leadership Program. Dr. Barry receives grant support 
through Massachusetts General Hospital from Healthwise, a nonprofit patient education and decision support organization. Dr. Davis is the CEO of ACP Decisions, a non-profit private foundation. JAT is a Founding Director of VitalTalk, a non-profit organization focused on clinician communication skills training, from which he receives no compensation. AV has a financial interest in ACP Decisions Nous, a non-profit organization developing ACP video decision support tools. His interests were reviewed and are managed by Massachusetts General Hospital and Partners HealthCare in accordance with their conflict of interest policies.

Patient and public involvement Patients and/or the public were not involved in the design, or conduct, or reporting, or dissemination plans of this research.

Patient consent for publication Not required.

Provenance and peer review Not commissioned; peer reviewed for ethical and funding approval prior to submission.

Open access This is an open access article distributed in accordance with the Creative Commons Attribution Non Commercial (CC BY-NC 4.0) license, which permits others to distribute, remix, adapt, build upon this work non-commercially, and license their derivative works on different terms, provided the original work is properly cited, appropriate credit is given, any changes made indicated, and the use is non-commercial. See: http://creativecommons.org/licenses/by-nc/4.0/.

ORCID iD

Joshua R Lakin http://orcid.org/0000-0002-7659-6512

\section{REFERENCES}

1 Berger NA, Savvides P, Koroukian SM, et al. Cancer in the elderly. Trans Am Clin Climatol Assoc 2006;117:147-55.

2 Howlader NA, Krapcho M, Miller D, et al. Seer cancer statistics review, 1975-2013, National cancer Institute.

3 Ahronheim JC, Morrison RS, Baskin SA, et al. Treatment of the dying in the acute care hospital. advanced dementia and metastatic cancer. Arch Intern Med 1996;156:2094-100.

4 Earle CC, Landrum MB, Souza JM, et al. Aggressiveness of cancer care near the end of life: is it a quality-of-care issue? JCO 2008;26:3860-6.

5 Earle CC, Neville BA, Landrum MB, et al. Trends in the aggressiveness of cancer care near the end of life. JCO 2004:22:315-21.

6 Aitken PV. Incorporating advance care planning into family practice [see comment]. Am Fam Physician 1999;59:605-14.

7 Tulsky JA. Improving quality of care for serious illness: findings and recommendations of the Institute of medicine report on dying in America. JAMA Intern Med 2015;175:840-1.

8 Mack JW, Weeks JC, Wright AA, et al. End-Of-Life discussions, goal attainment, and distress at the end of life: predictors and outcomes of receipt of care consistent with preferences. J Clin Oncol 2010;28:1203-8.

9 Wright AAet al. Associations between end-of-life discussions, patient mental health, medical care near death, and caregiver bereavement adjustment. JAMA 2008;300:1665-73.

10 Bernacki RE, Block SD. American College of physicians high value care task $\mathrm{f}$. communication about serious illness care goals: a review and synthesis of best practices. JAMA Intern Med 2014:174:1994-2003.

11 Mitchell SL, Kiely DK, Hamel MB. Dying with advanced dementia in the nursing home. Arch Intern Med 2004;164:321-6.

12 Mitchell SL, Teno JM, Kiely DK, et al. The clinical course of advanced dementia. N Engl J Med 2009;361:1529-38.

13 Thorne SE, Bultz BD, Baile WF, et al. Is there a cost to poor communication in cancer care?: a critical review of the literature. Psychooncology 2005;14:875-84.

14 Office of the Assistant Secretary for Planning and Evalation. U.S. Department of Health and Human Services. Advance directives and advance care planning: report to Congress 2008.

15 Heyland DKet al. Failure to engage hospitalized elderly patients and their families in advance care planning. JAMA Intern Med 2013;173:778-87.

16 Emanuel LL, Barry MJ, Stoeckle JD, et al. Advance directives for medical care--a case for greater use. N Engl J Med 1991;324:889-95.

17 Emanuel LL, von Gunten CF, Ferris FD. Advance care planning. Arch Fam Med 2000;9:1181-7.

18 Morrison RS, Meier DE. High Rates of Advance Care Planning in New York City's Elderly Population. Arch Intern Med 2004;164:2421-6.
19 Billings JA. The need for safeguards in advance care planning. J Gen Intern Med 2012;27:595-600.

20 Billings JA, Bernacki R. Strategic targeting of advance care planning interventions: the Goldilocks phenomenon. JAMA Intern Med 2014;174:620-4

21 Fagerlin A, Schneider CE. Enough. The failure of the living will. Hastings Cent Rep 2004;34:30-42.

22 Lakin JR, Block SD, Billings JA, et al. Improving communication about serious illness in primary care. JAMA Intern Med 2016;176:1380-7.

23 Loewenstein G. Hot-cold empathy gaps and medical decision making. Health Psychol 2005;24:S49-56.

24 Winter L, Parks SM, Diamond JJ. Ask a different question, get a different answer: why living wills are poor guides to care preferences at the end of life. J Palliat Med 2010;13:567-72.

25 Patel K, Janssen DJA, Curtis JR. Advance care planning in COPD. Respirology 2012;17:72-8.

26 Davison SN, Simpson C. Hope and advance care planning in patients with end stage renal disease: qualitative interview study. BMJ 2006:333:886.

27 Fischer GS, Tulsky JA, Rose MR, et al. Patient knowledge and physician predictions of treatment preferences after discussion of advance directives. J Gen Intern Med 1998;13:447-54.

28 Quill TE. Perspectives on care at the close of life. Initiating end-of-life discussions with seriously ill patients: addressing the "elephant in the room". JAMA 2000;284:2502-7.

29 Sharman SJ, Garry M, Jacobson JA, et al. False memories for endof-life decisions. Health Psychol 2008;27:291-6.

30 Friedrichsen MJ, Strang PM, Carlsson ME. Breaking bad news in the transition from curative to palliative cancer care-patient's view of the doctor giving the information. Support Care Cancer 2000;8:472-8.

31 Detmar SB, Aaronson NK, Wever LD, et al. How are you feeling? who wants to know? patients' and oncologists' preferences for discussing health-related quality-of-life issues. J Clin Oncol 2000;18:3295-301.

32 Wenrich MD, Curtis JR, Shannon SE, et al. Communicating with dying patients within the spectrum of medical care from terminal diagnosis to death. Arch Intern Med 2001;161:868-74.

33 Butow PN, Kazemi JN, Beeney LJ, et al. When the diagnosis is cancer: patient communication experiences and preferences. Cancer 1996:77:2630-7.

34 Clark RE, LaBeff EE. Death telling: managing the delivery of bad news. J Health Soc Behav 1982;23:366-80.

35 Eden OB, Black I, MacKinlay GA, et al. Communication with parents of children with cancer. Palliat Med 1994;8:105-14.

36 Ford S, Fallowfield L, Lewis S. Can oncologists detect distress in their out-patients and how satisfied are they with their performance during bad news consultations? Br J Cancer 1994;70:767-70.

37 Ford S, Fallowfield L, Lewis S. Doctor-Patient interactions in oncology. Soc Sci Med 1996;42:1511-9.

38 Ptacek JT, Eberhardt TL. Breaking bad news. A review of the literature. JAMA 1996;276:496-502.

39 Bakitas M, Lyons KD, Hegel MT, et al. Effects of a palliative care intervention on clinical outcomes in patients with advanced cancer: the project enable II randomized controlled trial. JAMA 2009;302:741-9.

40 Bakitas MA, Tosteson TD, Li Z, et al. Early versus delayed initiation of concurrent palliative oncology care: patient outcomes in the enable III randomized controlled trial. JCO 2015;33:1438-45.

41 Hanson LC, Zimmerman S, Song MK, et al. Effect of the goals of care intervention for advanced dementia: a randomized clinical trial. JAMA Intern Med 2017:177:24-31.

42 Temel JS, Greer JA, Muzikansky A, et al. Early palliative care for patients with metastatic Non-Small-Cell lung cancer. N Engl J Med 2010;363:733-42.

43 Thorpe KE, Zwarenstein M, Oxman AD, et al. A pragmaticexplanatory continuum indicator summary (Precis): a tool to help trial designers. J Clin Epidemiol 2009;62:464-75.

44 Ford I, Norrie J, Trials P. Pragmatic trials. N Engl J Med Overseas Ed 2016;375:454-63.

45 Chan A-W, Tetzlaff JM, Altman DG, et al. Spirit 2013 statement: defining standard protocol items for clinical trials. Ann Intern Med 2013;158:200-7.

46 Back ALet al. Efficacy of communication skills training for giving bad news and discussing transitions to palliative care. Arch Intern Med 2007;167:453-60.

47 Back AL, Arnold RM, Baile WF, et al. Faculty development to change the paradigm of communication skills teaching in oncology. J Clin Oncol 2009;27:1137-41.

48 Back AL, Arnold RM, Tulsky JA, et al. Teaching communication skills to medical oncology fellows. J Clin Oncol 2003;21:2433-6. 
49 Clayton JM, Adler JL, O'Callaghan A, et al. Intensive communication skills teaching for specialist training in palliative medicine: development and evaluation of an experiential workshop. J Palliat Med 2012;15:585-91.

50 Clayton JM, Butow PN, Waters A, et al. Evaluation of a novel individualised communication-skills training intervention to improve doctors' confidence and skills in end-of-life communication. Palliat Med 2013;27:236-43.

51 Fryer-Edwards K, Arnold RM, Baile W, et al. Reflective teaching practices: an approach to teaching communication skills in a smallgroup setting. Acad Med 2006;81:638-44.

52 Tulsky JA, Arnold RM, Alexander SC, et al. Enhancing communication between oncologists and patients with a computerbased training program. Ann Intern Med 2011;155:593-601.

53 Cohen SM, Volandes AE, Shaffer ML, et al. Concordance between proxy level of care preference and advance directives among nursing home residents with advanced dementia: a cluster randomized clinical trial. J Pain Symptom Manage 2019;57:37-46.

54 Deep KS, Hunter A, Murphy K, et al. "It helps me see with my heart": How video informs patients' rationale for decisions about future care in advanced dementia. Patient Educ Couns 2010;81:229-34.

55 El-Jawahri A, Paasche-Orlow MK, Matlock D, et al. Randomized, controlled trial of an advance care planning video decision support tool for patients with advanced heart failure. Circulation 2016;134:52-60.

56 El-Jawahri A, Podgurski LM, Eichler AF, et al. Use of video to facilitate end-of-life discussions with patients with cancer: a randomized controlled trial. JCO 2010;28:305-10.

57 Epstein AS, Volandes AE, Chen LY, et al. A randomized controlled trial of a cardiopulmonary resuscitation video in advance care planning for progressive pancreas and hepatobiliary cancer patients. J Palliat Med 2013;16:623-31.

58 McCannon JB, O'Donnell WJ, Thompson BT, et al. Augmenting communication and decision making in the intensive care unit with a cardiopulmonary resuscitation video decision support tool: a temporal intervention study. J Palliat Med 2012;15:1382-7.

59 Quintiliani LM, Murphy JE, Buitron de la Vega P, et al. Feasibility and patient perceptions of video Declarations regarding end-of-life decisions by hospitalized patients. J Palliat Med 2018;21:766-72.

60 Volandes AE, Ariza M, Abbo ED, et al. Overcoming educational barriers for advance care planning in Latinos with video images. $J$ Palliat Med 2008;11:700-6.

61 Volandes AE, Barry MJ, Chang Y, et al. Improving decision making at the end of life with video images. Med Decis Making 2010;30:29-34.

62 Volandes AE, Brandeis GH, Davis AD, et al. A randomized controlled trial of a goals-of-care video for elderly patients admitted to skilled nursing facilities. J Palliat Med 2012;15:805-11.

63 Volandes AE, Ferguson LA, Davis AD, et al. Assessing end-oflife preferences for advanced dementia in rural patients using an educational video: a randomized controlled trial. J Palliat Med 2011;14:169-77.

64 Volandes AEet al. Using video images of dementia in advance care planning. Arch Intern Med 2007;167:828-33.

65 Volandes AE, Levin TT, Slovin S, et al. Augmenting advance care planning in poor prognosis cancer with a video decision aid: a preintervention-postintervention study. Cancer 2012;118:4331-8.

66 Volandes AE, Mitchell SL, Gillick MR, et al. Using video images to improve the accuracy of surrogate decision-making: a randomized controlled trial. J Am Med Dir Assoc 2009;10:575-80.

67 Volandes AE, Paasche-Orlow M, Gillick MR, et al. Health literacy not race predicts end-of-life care preferences. J Palliat Med 2008:11:754-62.
68 Volandes AE, Paasche-Orlow MK, Barry MJ, et al. Video decision support tool for advance care planning in dementia: randomised controlled trial. BMJ 2009;338:b2159.

69 Volandes AE, Paasche-Orlow MK, Davis AD, et al. Use of Video Decision Aids to Promote Advance Care Planning in Hilo, Hawai'i. $J$ Gen Intern Med 2016;31:1035-40.

70 Volandes AE, Paasche-Orlow MK, Mitchell SL, et al. Randomized controlled trial of a video decision support tool for cardiopulmonary resuscitation decision making in advanced cancer. JCO 2013;31:380-6.

71 El Jawahri A, Temel JS, Ramachandran KJ, et al. A randomized controlled trial of a CPR video decision support tool for seriously ill hospitalized patients with advanced cancer. ASCO annual meeting 2015.

72 El-Jawahri A, Mitchell SL, Paasche-Orlow MK, et al. A randomized controlled trial of a CPR and intubation video decision support tool for hospitalized patients. J Gen Intern Med 2015;30:1071-80.

73 Washington State Health Care Authority. Patient decision AIDS Available: https://www.hca.wa.gov/about-hca/healthier-washington/ patient-decision-aids-pdas [Accessed 11 May 2020].

74 Dyer N, Sorra JS, Smith SA, et al. Psychometric properties of the consumer assessment of healthcare providers and systems (CAHPS $尺$ ) clinician and group adult visit survey. Med Care 2012;50:S28-34.

75 Mukherjee S, Rodriguez HP, Elliott MN, et al. Modern psychometric methods for estimating physician performance on the clinician and group CAHPS $₫$ survey. Health Serv Outcomes Res Method 2013;13:109-23.

76 Holmes-Rovner M, Kroll J, Schmitt N, et al. Patient Satisfaction with Health Care Decisions:The Satisfaction with Decision Scale 1996;16:58-64.

77 Brehaut JC, O'Connor AM, Wood TJ, et al. Validation of a decision regret scale. Med Decis Making 2003;23:281-92.

78 Goel V, Sawka CA, Thiel EC, et al. Randomized trial of a patient decision aid for choice of surgical treatment for breast cancer. Med Decis Making 2001;21:1-6.

79 Hassett MJ, Ritzwoller DP, Taback N, et al. Validating billing/ encounter codes as indicators of lung, colorectal, breast, and prostate cancer recurrence using 2 large contemporary cohorts. Med Care 2014;52:e65-73.

80 Lindvall C, Lilley EJ, Zupanc SN, et al. Natural language processing to assess end-of-life quality indicators in cancer patients receiving palliative surgery. J Palliat Med 2019;22:183-7.

81 Poort $\mathrm{H}$, Zupanc SN, Leiter RE, et al. Documentation of palliative and end-of-life care process measures among young adults who died of cancer: a natural language processing approach. $J$ Adolesc Young Adult Oncol 2020;9:100-4.

82 Udelsman BV, Lilley EJ, Qadan M, et al. Deficits in the palliative care process measures in patients with advanced pancreatic cancer undergoing operative and invasive Nonoperative palliative procedures. Ann Surg Oncol 2019;26:4204-12.

83 Wright A. REDCap: a tool for the electronic capture of research data J Electron Resour Med Libr 2016;13:197-201.

84 Pugno PA. Advance directives in the primary care setting. Wien Klin Wochenschr 2004:116:417-9.

85 Hughes JP, Granston TS, Heagerty PJ. Current issues in the design and analysis of stepped wedge trials. Contemp Clin Trials 2015;45:55-60.

86 Hooper R, Bourke L. Cluster randomised trials with repeated cross sections: alternatives to parallel group designs. BMJ 2015:350:h2925.

87 Hooper R, Teerenstra S, de Hoop E, et al. Sample size calculation for stepped wedge and other longitudinal cluster randomised trials. Stat Med 2016;35:4718-28. 